\title{
Macroscopic superpositions in Bose-Josephson junctions: controlling decoherence due to atom losses
}

\author{
K. Pawlowski, ${ }^{1,2,3}$ D. Spehner, ${ }^{4,5}$, , A. Minguzzi, ${ }^{5}$ and G. Ferrini ${ }^{6}$ \\ ${ }^{1}$ Center for Theoretical Physics PAN, Al. Lotników 32/46, 02-668 Warsaw, Poland \\ 25. Physikalisches Institut, Universität Stuttgart, \\ Pfaffenwaldring 57, D-70569 Stuttgart, Germany \\ ${ }^{3}$ Laboratoire Kastler Brossel, Ecole Normale Supérieure, 45, rue d'Ulm, 75231 Paris, France \\ ${ }^{4}$ Université Grenoble 1 and CNRS, Institut Fourier UMR5582, B.P. 74, 38402 Saint Martin d'Hères, France \\ ${ }^{5}$ Université Grenoble 1 and CNRS, Laboratoire de Physique et Modélisation \\ des Milieux Condensés UMR5493, B.P. 166, 38042 Grenoble, France \\ ${ }^{6}$ Laboratoire Kastler Brossel, Université Pierre et Marie Curie, 4 place de Jussieu, 75005 Paris, France
}

(Dated: September 17, 2018)

\begin{abstract}
We study how macroscopic superpositions of coherent states produced by the nondissipative dynamics of binary mixtures of ultracold atoms are affected by atom losses. We identify different decoherence scenarios for symmetric or asymmetric loss rates and interaction energies in the two modes. In the symmetric case the quantum coherence in the superposition is lost after a single loss event. By tuning appropriately the energies we show that the superposition can be protected, leading to quantum correlations useful for atom interferometry even after many loss events.

PACS numbers: 03.75Gg, 42.50.Lc, 03.75.Lm, 03.75.Mn
\end{abstract}

\section{INTRODUCTION}

In large systems, macroscopic superpositions of quantum states have extremely small decoherence times, making them impossible to observe [1]. The largest superpositions generated so far are superpositions of coherent states (CSs) of a cavity field, and their progressive transformation into statistical mixtures as time evolves has been observed [2]. The first proposal to generate a macroscopic superposition of CSs (MSCSs) with light consisted of sending photons through a medium presenting a strong Kerr nonlinearity [3]. In such media, the dynamical phases of Fock states are nonlinear in the photon number, thus the phase of an initial CS is split. In metastable vapors of ultracold bosonic atoms, interactions between atoms lead to similar nonlinearities, whose strength can be tuned experimentally by using Feshbach resonances [4, 5]. By trapping optically the condensed atoms in a double-well potential one realizes an external Bose-Josephson junction (BJJ); an internal BJJ is obtained by trapping in a single well atoms in two distinct hyperfine states coupled by a resonant field. In analogy with light, the nonlinear dynamics generates MSCSs after a sudden quench to zero of the tunnel amplitude (for external BJJs) or a switch off of the coupling field (for internal BJJs) [6, 7]. Whereas MSCSs of light are presumably destroyed after a single photon loss [2], the situation is less clear for atoms. Up to now only squeezed states - which are produced at earlier times than MSCSs - have been observed in BJJs with a few hundreds of atoms [8 -10]. In order to know if future experiments could produce MSCSs in BJJs, it is desirable to study how robust they are with respect to decoherence.
Decoherence effects due to atom losses and phase noise on squeezed states have been analyzed in detail [11 13]. For what concerns MSCSs, only decoherence caused by noise due to photon scattering [14] and phase noise [15] has been studied so far. Under current experimental conditions the first noise is negligible and the second one can be reduced by using spin echo [10, 16]; in contrast, atom losses are unavoidable.

In this article, we investigate whether a macroscopic superposition can be formed in a BJJ even in the presence of atom losses. We are primarily interested in internal Bose-Josephson junctions, such as those studied experimentally in $[9,10]$. We focus on two-body losses, due to scattering of two atoms in the magnetic trap which changes their spin and gives them enough kinetic energy to be ejected from the trap. These loss processes are particularly detrimental in the experiments of Refs. [9, 10]. Our analysis, however, also applies to one- and threebody losses [17]. We first analyze the dynamics of a lossy BJJ from the point of view of state conditioning, assuming that the total number $\hat{N}$ of condensed atoms can be measured precisely, both initially and at the MSCSs formation time $t_{q}$. We study how much coherence is destroyed by a single loss event occurring at a random time between 0 and $t_{q}$. We find quite different answers depending on the degree of asymmetry between the loss rates and interaction energies in the two modes of the junction. Finally, we show that for strongly asymmetric losses one can protect the coherence of the MSCSs by suitably tuning the interaction energies, even after many loss events and in the absence of measurement of the atom number.

* Dominique.Spehner@ujf-grenoble.fr 


\section{QUENCHED DYNAMICS IN BOSE-JOSEPHSON JUNCTIONS IN THE PRESENCE OF ATOM LOSSES}

We consider an internal BJJ in the quantum regime. Initially, $N_{0}$ atoms are all in the same single-particle state which is a symmetric superposition of the two internal states. This corresponds to the ground state in the regime where tunneling dominates interactions, described by a spin coherent state

$$
\begin{aligned}
& |\psi(0)\rangle=\left|N_{0} ; \theta, \phi\right\rangle \\
& =\sum_{n_{1}=0}^{N_{0}}\left(\begin{array}{c}
N_{0} \\
n_{1}
\end{array}\right)^{1 / 2} \frac{\left(e^{-\mathrm{i} \phi} \tan (\theta / 2)\right)^{n_{1}}}{\left[1+\tan ^{2}(\theta / 2)\right]^{N_{0} / 2}}\left|n_{1}, N_{0}-n_{1}\right\rangle
\end{aligned}
$$

with $\theta=\pi / 2$ and $\phi=0$. Here $\left|n_{1}, n_{2}\right\rangle$ is the joint eigenstate of the number operators $\hat{n}_{i}$ in the mode $i=1,2$ (Fock state). The dynamics following a sudden quench of the coupling (tunnel energy) to zero is given by the two-mode Bose-Hubbard Hamiltonian [18]

$$
\hat{H}_{0}=\sum_{i=1,2}\left(E_{i} \hat{n}_{i}+\frac{U_{i}}{2} \hat{n}_{i}\left(\hat{n}_{i}-1\right)\right)+U_{12} \hat{n}_{1} \hat{n}_{2},
$$

where $E_{i}$ and $U_{i}$ are the internal energy and the interaction energy between two atoms in the same mode $i$, respectively, and $U_{12}$ is the inter-mode interaction energy. Setting $\hat{n}_{2}=N_{0}-\hat{n}_{1}$, the interactions in Eq. (2) sum up to a non-linear term $\chi \hat{n}_{1}^{2}$, with $\chi=\left(U_{1}+U_{2}-\right.$ $\left.2 U_{12}\right) / 2$. The time-evolved state at time $t_{q}=\pi /|\chi q|$ is a superposition of CSs, $\left|\psi^{(0)}\left(t_{q}\right)\right\rangle=e^{-\mathrm{i} t_{q} \hat{H}_{0}}|\psi(0)\rangle=$ $\sum_{k=0}^{q-1} c_{k}\left|N_{0} ; \frac{\pi}{2}, \phi_{k}\right\rangle$, with $\phi_{k+1}-\phi_{k}=2 \pi / q$ and $\left|c_{k}\right|=$ $q^{-1 / 2}$ [3].

In the presence of two-body losses the markovian master equation for the density matrix $\hat{\rho}(t)$ of the condensed atoms reads [19, 20] (setting $\hbar=1)$

$$
\begin{aligned}
& \frac{\mathrm{d} \hat{\rho}(t)}{\mathrm{d} t}=-\mathrm{i}\left[\hat{H}_{0}, \hat{\rho}\right]+\sum_{i=1,2} \gamma_{i}\left(\hat{a}_{i}^{2} \hat{\rho}\left(\hat{a}_{i}^{2}\right)^{\dagger}\right. \\
& \left.-\frac{1}{2}\left\{\hat{n}_{i}\left(\hat{n}_{i}-1\right), \hat{\rho}\right\}\right)+\gamma_{12}\left(\hat{a}_{1} \hat{a}_{2} \hat{\rho} \hat{a}_{1}^{\dagger} \hat{a}_{2}^{\dagger}-\frac{1}{2}\left\{\hat{n}_{1} \hat{n}_{2}, \hat{\rho}\right\}\right)
\end{aligned}
$$

where $\gamma_{i}$ and $\gamma_{12}$ are the loss rates of two atoms in the same mode $i$ and of one atom in each mode, respectively, and $\{\cdot, \cdot\}$ denotes the anticommutator. Since Eq. (3) does not couple subspaces with different total atom numbers $\hat{N}=\hat{n}_{1}+\hat{n}_{2}$ and $\hat{N}=N_{0}$ initially, at all times $\hat{\rho}(t)$ has the block structure $\hat{\rho}(t)=\sum_{N=0}^{N_{0}} w_{N}(t) \hat{\rho}_{N}(t)$, where $w_{N}(t)$ is the probability to have $N$ atoms at time $t$ and $\hat{\rho}_{N}(t)$ is the corresponding conditional state. This decomposition is naturally accounted for by quantum trajectories $t \mapsto\left|\psi_{J}(t)\right\rangle=\left|\widetilde{\psi}_{J}(t)\right\rangle /\left\|\widetilde{\psi}_{J}(t)\right\|,\left|\widetilde{\psi}_{J}(t)\right\rangle$ being the unnormalized wave function when $J$ loss events occur at times $0 \leq s_{1} \leq s_{2} \leq \cdots \leq s_{J} \leq t$ in the channels $m_{1}, \cdots, m_{J}$ [2, 21, 22],

$$
\begin{aligned}
\left|\widetilde{\psi}_{J}(t)\right\rangle= & e^{-\mathrm{i}\left(t-s_{J}\right) \hat{H}_{\text {eff }}} \hat{M}_{m_{J}} e^{-\mathrm{i}\left(s_{J}-s_{J-1}\right) \hat{H}_{\text {eff }}} \hat{M}_{m_{J-1}} \cdots \\
& \cdots e^{-\mathrm{i} \hat{H}_{\text {eff }}\left(s_{2}-s_{1}\right)} \hat{M}_{m_{1}} e^{-\mathrm{i} s_{1} \hat{H}_{\text {eff }}}|\psi(0)\rangle
\end{aligned}
$$

For two-body losses one has three loss channels $m=1,2$, and 12. The corresponding jump operators are $\hat{M}_{1}=\hat{a}_{1}^{2}$, $\hat{M}_{2}=\hat{a}_{2}^{2}$, and $\hat{M}_{12}=\hat{a}_{1} \hat{a}_{2}$. The dynamics between loss events is given by the effective Hamiltonian $\hat{H}_{\text {eff }}=\hat{H}_{0}-$ $\mathrm{i} \hat{D}$. The damping operator

$$
\hat{D}=\frac{1}{2}\left(\sum_{i=1,2} \gamma_{i} \hat{n}_{i}\left(\hat{n}_{i}-1\right)+\gamma_{12} \hat{n}_{1} \hat{n}_{2}\right)
$$

describes the gain of information on the system resulting from the knowledge that no loss occurred. The conditional state $\hat{\rho}_{N}(t)$ after a detection of $N=N_{0}-2 J$ atoms at time $t$ is obtained by averaging $\left|\psi_{J}(t)\right\rangle\left\langle\psi_{J}(t)\right|$ over the $J$ jump times $s_{K}$ and channels $m_{K}$,

$$
\begin{aligned}
\widetilde{\rho}_{N}(t) \equiv & w_{N}(t) \hat{\rho}_{N}(t)=\sum_{m_{1}, \cdots, m_{J}} \int_{0 \leq s_{1} \leq \cdots \leq s_{J} \leq t} \mathrm{~d} s_{1} \ldots \mathrm{d} s_{J} \\
& \times p_{m_{1}, \ldots, m_{J}}^{(t)}\left(s_{1}, \ldots, s_{J} ; J\right)\left|\psi_{J}(t)\right\rangle\left\langle\psi_{J}(t)\right|
\end{aligned}
$$

where $p_{m_{1}, \ldots, m_{J}}^{(t)}\left(s_{1}, \ldots, s_{J} ; J\right)=\gamma_{m_{1}} \ldots \gamma_{m_{J}}\left\|\widetilde{\psi}_{J}(t)\right\|^{2}$ is the joint distribution of the $s_{K}, m_{K}$, and $J$ [22]. By further summing over the number of jumps $J$ one gets the total density matrix $\hat{\rho}(t)=\sum_{N=0}^{N_{0}} w_{N}(t) \hat{\rho}_{N}(t)$ which is a solution of Eq. (3). In order to understand the effect of losses we analyze separately each $N$-atom sectors.

\section{CONDITIONAL STATES AND THEIR QUANTUM CORRELATIONS}

\section{A. Density matrix in the subspace with $N_{0}$ atoms}

When no loss occurs in the time interval $[0, t]$, from Eqs. (4) and (5) we obtain the unnormalized conditional state $\widetilde{\rho}_{N_{0}}^{\text {(noloss) }}(t)=\left|\widetilde{\psi}_{0}(t)\right\rangle\left\langle\widetilde{\psi}_{0}(t)\right|$ in the Fock basis,

$$
\begin{aligned}
& \left\langle n_{1}, n_{2}\left|\widetilde{\rho}_{N_{0}}^{(\text {noloss })}(t)\right| n_{1}^{\prime}, n_{2}^{\prime}\right\rangle \\
& \quad=e^{-t\left[d_{N_{0}}\left(n_{1}\right)+d_{N_{0}}\left(n_{1}^{\prime}\right)\right]}\left\langle n_{1}, n_{2}\left|\hat{\rho}^{(0)}(t)\right| n_{1}^{\prime}, n_{2}^{\prime}\right\rangle,
\end{aligned}
$$

where $\hat{\rho}^{(0)}(t)=\left|\psi^{(0)}(t)\right\rangle\left\langle\psi^{(0)}(t)\right|$ is the lossless density matrix and

$$
d_{N_{0}}\left(n_{1}\right)=\frac{1}{2}\left(\gamma_{1}+\gamma_{2}-\gamma_{12}\right)\left(n_{1}-\bar{n}_{1}\right)^{2}
$$

up to an irrelevant constant, with $2 \bar{n}_{1}=\left[\gamma_{1}-\gamma_{2}+\right.$ $\left.N_{0}\left(2 \gamma_{2}-\gamma_{12}\right)\right] /\left(\gamma_{1}+\gamma_{2}-\gamma_{12}\right)$. For $N_{0} \gg 1$ the matrix elements of $\hat{\rho}^{(0)}(t)$ have Gaussian moduli peaked at $\left(n_{1}, n_{1}^{\prime}\right)=\left(N_{0} / 2, N_{0} / 2\right)$ with a width $\sim \sqrt{N_{0}}$. For symmetric loss rates $\gamma_{1}=\gamma_{2}$ and $\gamma_{12}=0$, this peak coincides with the center of the Gaussian damping factor in Eq. (7). Thus the MSCSs formed at time $t_{q}$ is affected by damping when $\gamma_{1} \gtrsim|\chi| q / N_{0}$. In contrast, for $\gamma_{2}=\gamma_{12}=0$ the damping factor is centered at $\left(n_{1}, n_{1}^{\prime}\right)=(1 / 2,1 / 2)$ and its effect on the MSCSs sets in at the much smaller rate $\gamma_{1} \approx|\chi| q / N_{0}^{2}$ 

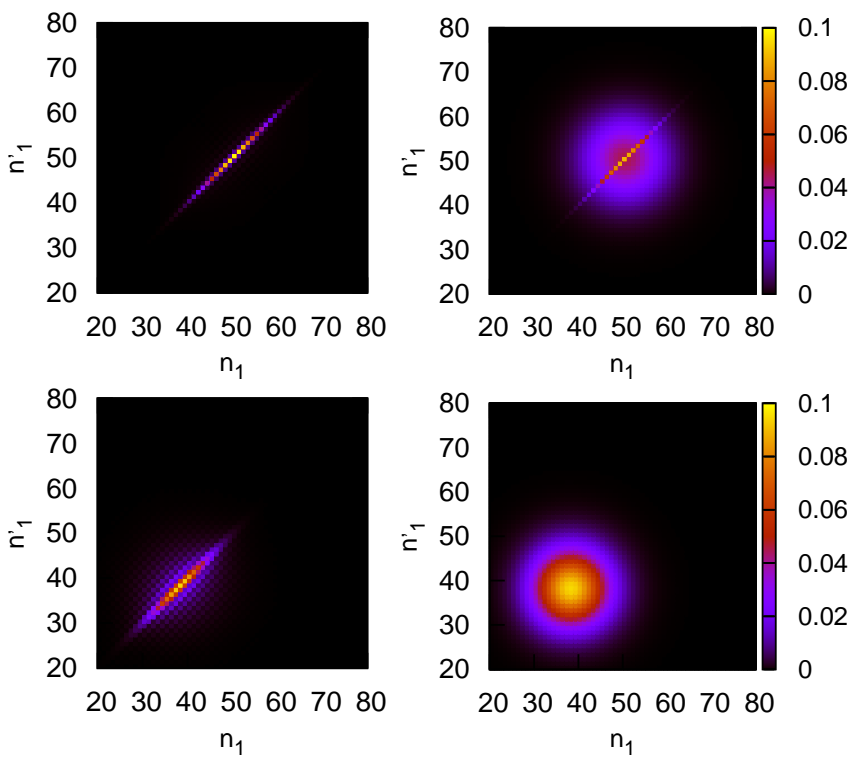

FIG. 1. (Color online) Modulus of the density matrix $\hat{\rho}_{N_{0}-2}\left(t_{2}\right)$ in the subspace with $N_{0}-2$ atoms in the Fock basis at time $t_{2}$, obtained by an exact diagonalization of Eq. (3). Upper panels: symmetric losses rates $\left(\gamma_{1}=\gamma_{2}=\chi /(200 \pi)\right)$; lower panels: asymmetric losses $\left(\gamma_{1}=4 \chi /(300 \pi), \gamma_{2}=0\right)$. Left column: symmetric energies $\left(\chi_{1}=-\chi_{2}=\chi\right)$; right column: asymmetric energies $\left(\chi_{1}=2 \chi, \chi_{2}=0\right)$. Other parameters: $\gamma_{12}=E_{i}=0, N_{0}=100$.

B. Density matrix in the subspace with $\left(N_{0}-2\right)$ atoms: Tuning the energies to protect the coherence

We focus now on trajectories having one loss event in channel $m$ at the random time $s \in[0, t]$. The instantaneous jump transforms a CS into a CS, $\hat{M}_{m}\left|N_{0} ; \theta, \phi\right\rangle \propto$ $\left|N_{0}-2 ; \theta, \phi\right\rangle$. This CS is rotated on the Bloch sphere by the evolution under the nonlinear effective Hamiltonian $\hat{H}_{\text {eff }}$ due to changes in energy and damping before and after the jump, yielding (see Appendix $\mathrm{A}$ )

$$
\left|\psi_{1}(t)\right\rangle \propto e^{-\mathrm{i} t \hat{H}_{\text {eff }}}\left|N_{0}-2 ; \theta_{m}(s), \phi_{m}(s)\right\rangle,
$$

where the random angles $\theta_{m}(s)=2 \arctan \left(e^{-s \delta_{m}}\right)$ and $\phi_{m}(s)=2 s \chi_{m}$ depend on the random loss time $s$, the interaction energies $\chi_{1}=U_{1}-U_{12}, \chi_{2}=-\left(U_{2}-U_{12}\right)$, $\chi_{12}=\left(U_{1}-U_{2}\right) / 2$, and the loss rate differences $\delta_{1}=$ $2 \gamma_{1}-\gamma_{12}, \delta_{2}=-\left(2 \gamma_{2}-\gamma_{12}\right)$, and $\delta_{12}=\gamma_{1}-\gamma_{2}$. Hence, apart from reducing $N$ and producing damping, atom losses are identical to external $\theta$ and $\phi$ noises rotating the state around the $z$ axis by a complex angle $\phi_{m}+$ $\mathrm{i} \ln \tan \left(\frac{\theta_{m}}{2}\right)$ [23]. These noises have fluctuations $\delta \theta_{m} \simeq$ $\left|\delta_{m}\right| \min \left\{t, \delta s_{m}\right\}$ and $\delta \phi_{m}=2\left|\chi_{m}\right| \min \left\{t, \delta s_{m}\right\}$, where $\delta s_{m}$ is the loss-time fluctuation and we assumed $\delta \theta_{m} \ll 1$.

Let us concentrate on the MSCS formation time $t=t_{q}$ and take $\gamma_{12}=0$. We first consider weak losses $\gamma_{m} \lesssim$ $q \chi / N_{0}$ and symmetric energies $U_{1}=U_{2}$. In this regime the $\theta$-noise is negligible since $\delta \theta_{m}$ is much smaller than the quantum fluctuations $\sim 1 / \sqrt{N_{0}}$ of a CS. In contrast, one has large $\phi$ fluctuations $\delta \phi_{m}=2 \pi / q$ in the two loss channels $m=1,2$, equal to the inter-component phase separation of the MSCSs (we use here $\chi_{1}=-\chi_{2}=\chi$ and $\delta s_{m}>t_{q}$ ). Such a strong $\phi$ noise has a relatively small effect on the coherences, albeit it transforms the corresponding statistical mixture of CSs into a mixture of Fock states (phase relaxation) [15]. Actually, in the absence of losses in the second mode $\left(\gamma_{2}=0\right)$, the off-diagonal elements of the conditional density matrix $\left\langle n_{1}, n_{2}\left|\hat{\rho}_{N_{0}-2}\left(t_{q}\right)\right| n_{1}^{\prime}, n_{2}^{\prime}\right\rangle$ are weakly affected by losses for $n_{1}^{\prime} \neq n_{1}$ modulo $q$, as shown in Appendix [C and in Fig. 1 (lower left panel). The global shift to values $n_{1}, n_{1}^{\prime}<N_{0} / 2$ is due to the Gaussian damping caused by $\hat{H}_{\text {eff }}$ in Eq. (9). For $\gamma_{1}=\gamma_{2}$, instead, $\hat{\rho}_{N_{0}-2}\left(t_{2}\right)$ is almost diagonal in the Fock basis, see the upper left panel in Fig.1. This is due to a cancellation (occurring only for $q=2$ ) when summing the contributions of the two channels (see Appendix C).

We now consider asymmetric energies $U_{1} \neq U_{2}$, still assuming weak losses $\gamma_{m} \lesssim q \chi / N_{0}$. In order to keep $t_{q}=$ $\pi /|\chi q|$ constant, we vary $\chi_{1}, \chi_{2}$ while fixing $2 \chi=\chi_{1}-\chi_{2}$. Interestingly, it is possible to protect one channel, say $m=1$, against $\phi$ noise by choosing $\chi_{1}=0$ and $\chi_{2}=-2 \chi$, at the expense of enlarging noise in the other channel. Then $\delta \phi_{1}=0$ and $\delta \phi_{2}=4 \pi / q$. If only the first channel loses atoms, the conditional state after a single loss event is then close to a MSCSs with $N_{0}-2$ atoms, apart from the damping described in Eq. (7), as seen in the lower right panel in Fig. 1. For symmetric losses, $\hat{\rho}_{N_{0}-2}\left(t_{q}\right)$ has also large off-diagonal elements in the Fock basis due to the probability $1 / 2$ of losing atoms in the protected channel (Fig. 1 upper right panel). Hence, the MSCSs can be protected by tuning the interaction energies so that $\chi_{i}=0$ in the mode $i$ with the highest loss rate $\gamma_{i}$. For ${ }^{87} \mathrm{Rb}$ atoms used in the experiment of Ref. [10], a magnetic field in resonance with one of the Feshbach peaks for $m=1,2$, or 12 must be applied in order to have a nonzero $\chi$ (actually, without magnetic field one has nearly $U_{1}=U_{2}=U_{12}$ ). Since two-body losses are mostly important in the upper internal level $m=2$, in order to better preserve the coherence, $U_{1}$ must be tuned such that $\chi_{1}=2 \chi \neq 0$. Let us remark that, although our results also apply to external BJJs, for such BJJs $U_{12}=0$ and thus one must tune the interaction energy $U_{i}$ to zero to switch-off phase noise in the well $i$. But the loss rate $\gamma_{i}$ depends on $U_{i}$ and this tuning actually decreases $\gamma_{i}$, so that the protection of the MSCSs is a trivial effect. In contrast, for internal BJJs choosing $U_{i} \simeq U_{12}$ does not decrease the loss rates, but it diminishes decoherence in the loss channel $i$ at weak losses.

Let us now turn to the intermediate loss rate regime. The $\phi$ noise decreases when increasing $\gamma_{m}$ since the losstime fluctuations $\delta s_{m}$ decrease. Indeed, we find $\delta s_{i} \approx$ $\left(2 \gamma_{i}+\gamma_{12}\right)^{-1} N_{0}^{-1}(i=1,2)$ for $N_{0} \gg 1$. Physically, at increasing $\gamma_{m}$ the loss has more chance to occur at small times, while for small $\gamma_{m}, s$ is equally distributed in $[0, t]$. Note, however, that the probability $w_{N_{0}-2}\left(t_{q}\right)$ of losing only two atoms decreases by increasing $\gamma_{m}$. 


\section{Density matrix in the subspaces with $\left(N_{0}-2 J\right)$ atoms}

The wavefunction $\left|\psi_{J}(t)\right\rangle$ after $J>1$ jumps is still given by Eq. (9) upon replacing $N_{0}-2$ by $N_{0}-2 J$ and the angles of the CS by $\phi^{(J)}$ and $\theta^{(J)}$ with

$\phi^{(J)}=\sum_{K=1}^{J} \phi_{m_{K}}\left(s_{K}\right), \tan \left(\frac{\theta^{(J)}}{2}\right)=\prod_{K=1}^{J} \tan \left(\frac{\theta_{m_{K}}\left(s_{K}\right)}{2}\right)$,

where $\phi_{m}(s)$ and $\theta_{m}(s)$ are the angles corresponding to the single loss event. Thus the aforementioned effects persist. For weak symmetric losses, though, keeping coherence by switching off phase noise in one channel is harder since the probability that all jumps occur in that channel decreases exponentially with $J$. This means that for many loss events our proposal for protecting MSCSs is only efficient for strongly asymmetric loss rates.

\section{TOTAL QUANTUM CORRELATIONS AND SUB-SHOT NOISE ATOM INTERFEROMETRY}

We now show that for strongly asymmetric losses not only the conditional states but also the quantum correlations in the full density matrix $\hat{\rho}\left(t_{q}\right)$ can be protected by tuning the interactions. We measure the amount of quantum correlations with the quantum Fisher information $F(\hat{\rho})$, related to the best achievable phase precision in a Mach-Zehnder interferometer using $\hat{\rho}$ as input state by $(\Delta \varphi)_{\text {best }}=1 / \sqrt{F(\hat{\rho})}[24]$. Hence $F(\hat{\rho})>\langle\hat{N}\rangle$ implies phase accuracy beyond the shot noise limit $(\Delta \varphi)_{\mathrm{SN}}=\langle\hat{N}\rangle^{-1 / 2}$ [25]. In our case $F_{\text {tot }}(t) \equiv F[\hat{\rho}(t)]=\sum_{N=0}^{N_{0}} w_{N}(t) F_{N}(t)$ where $F_{N}(t)$ is the Fisher information of the conditional state $\hat{\rho}_{N}(t)$ in the subspace with $N$ atoms. The latter is given by $F_{N}=2 \sum_{k, l} \frac{\left(p_{k}-p_{l}\right)^{2}}{p_{k}+p_{l}}\left|\left\langle k\left|\hat{J}_{\mathbf{n}}\right| l\right\rangle\right|^{2}$ where $\hat{\rho}_{N}|l\rangle=p_{l}|l\rangle$ and $\hat{J}_{\mathbf{n}}$ is the angular momentum operator in the direction n. We optimize $F_{\text {tot }}(t)$ over all directions $\mathbf{n}$ of the interferometer. In a lossless BJJ, the two-component superposition has the highest possible value $F_{\text {tot }}\left(t_{2}\right)=N_{0}^{2}$, nearly twice larger than that of MSCSs with $q>2$ components [26, 27].

Figure 2 shows the Fisher information $F_{N}\left(t_{2}\right)$ in the subspace with $N=N_{0}-2$ atoms, corresponding to the state conditioned to a single loss event. For symmetric energies, the low values of $F_{N}$ at small symmetric loss rates are direct consequences of the cancellation among channels (see Appendix C). For $\gamma_{2}=0$ much larger values are found, showing that the aforementioned non-vanishing inter-component coherences carry useful quantum correlations. At intermediate rates, $F_{N}\left(t_{2}\right)$ increases and reaches a maximum as a result of the reduced phase noise when $\gamma_{1}=\gamma_{2}$ (note that this peak will not be seen on $F_{\text {tot }}\left(t_{2}\right)$ because of the rapid decay of the probability $w_{N_{0}-2}(t)$ by increasing $\left.\gamma_{m}\right)$. This reduction is clearly seen on the Husimi distributions

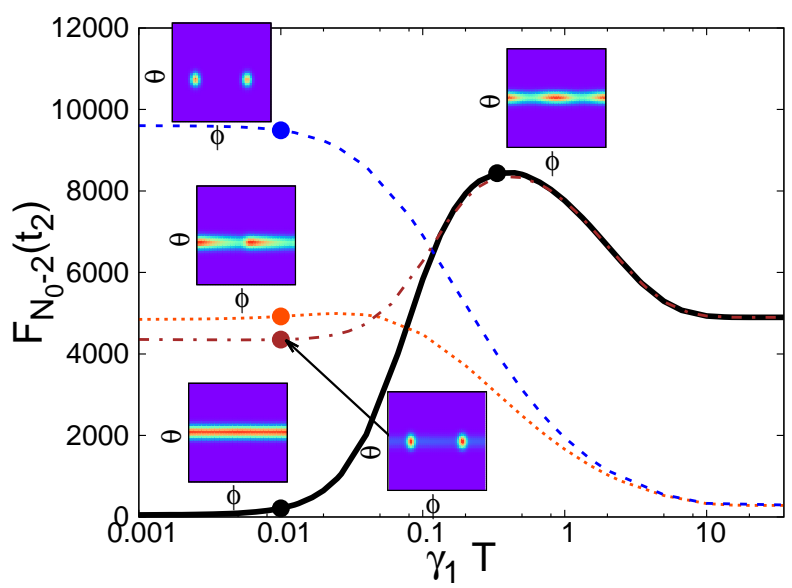

FIG. 2. (Color online) Fisher information in the subspace with $N_{0}-2$ atoms as a function of the loss rate $\gamma_{1}$ (in semilogarithmic scale) at time $t_{2}=T / 4$, with $T=2 \pi / \chi$. Solid black line: $\gamma_{1}=\gamma_{2}, \chi_{1}=-\chi_{2}=\chi$; dot-dashed brown line: $\gamma_{1}=\gamma_{2}$, $\chi_{1}=0, \chi_{2}=-2 \chi$; dotted red line: $\gamma_{2}=0, \chi_{1}=-\chi_{2}=\chi$; dashed blue line: $\gamma_{2}=\chi_{1}=0, \chi_{2}=-2 \chi$. The optimization over the interferometer directions is done independently in the $\left(N_{0}-2\right)$ subspace. Insets: Husimi functions for the values of $\gamma_{i}$ and $\chi_{i}$ corresponding to the circles on the curves. Other parameters as in Fig. 1

$Q_{N}(\theta, \phi)=\frac{1}{\pi}\left\langle N ; \theta, \phi\left|\hat{\rho}_{N}\left(t_{2}\right)\right| N ; \theta, \phi\right\rangle$ which display a flat profile for small losses and two emerging peaks for larger losses (insets in Fig. 2). For asymmetric losses $\gamma_{2}=0$, by choosing $\chi_{1}=0$ we have $F_{N}\left(t_{2}\right) \rightarrow N^{2}$ in the small loss limit and the Husimi function has two peaks at the CS phases $\phi= \pm \pi / 2$, in agreement with our prediction that the conditional state converges to a two-component superposition; at intermediate rates $F_{N}\left(t_{2}\right)$ decreases with $\gamma_{1}$ because the Gaussian damping compensates phase noise reduction. At large losses this damping transforms $\hat{\rho}_{N_{0}-2}\left(t_{2}\right)$ into a superposition of Fock states with $n_{1}=0$ or 1 atoms in the first mode [17]. For symmetric losses and even $N_{0}, \hat{\rho}_{N_{0}-2}\left(t_{2}\right)$ is transformed instead into the Fock state $\left|\frac{N}{2}, \frac{N}{2}\right\rangle$ having a larger Fisher information $\sim N^{2} / 2$.

Our main result is presented in Fig. 3, which displays the total Fisher information $F_{\text {tot }}(t)$ obtained from an exact diagonalization of Eq. (3) for various rates and energies, keeping the same value of $\chi$ and of the mean number of atoms at time $t_{2}$ (see the inset). We find that for $\gamma_{2}=0$, tuning the energies has a strong effect on $F_{\text {tot }}(t)$ at times $\chi t \gtrsim N_{0}^{-1 / 2}$, yielding to larger Fisher informations than for squeezed states. Quantum correlations in the total density matrix $\hat{\rho}(t)$ are then preserved even after the loss of $20 \%$ of atoms.

\section{CONCLUSIONS}

We have shown that the interplay of atom losses and interactions in BJJs leads to different decoherence sce- 


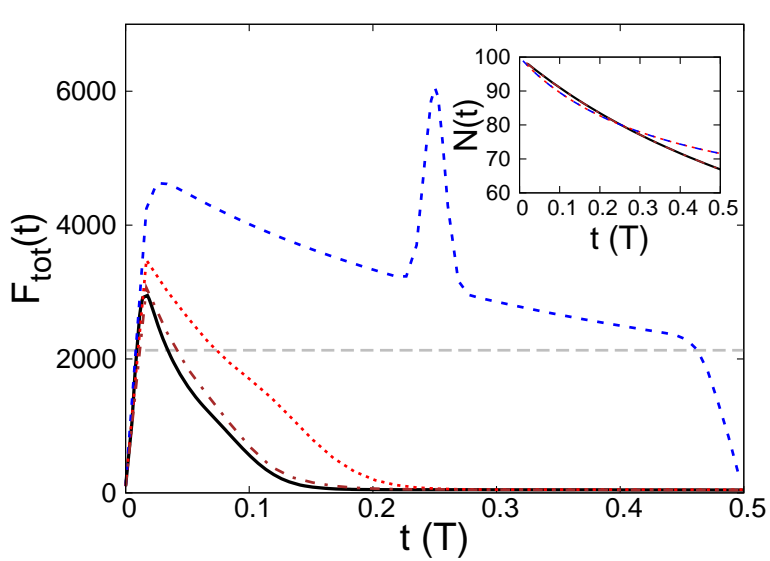

FIG. 3. (Color online) Total quantum Fisher information $F_{\text {tot }}(t)$ vs time $t$ (in units of $T$ ). From top to bottom: $\gamma_{1} T=8 / 300, \gamma_{2}=\chi_{1}=0, \chi_{2}=-2 \chi$ (blue dashed line); $\gamma_{1} T=8 / 300, \gamma_{2}=0, \chi_{1}=-\chi_{2}=\chi$ (red dotted line); $\gamma_{1} T=\gamma_{2} T=1 / 100, \chi_{1}=0, \chi_{2}=-2 \chi$ (brown dot-dashed line); $\gamma_{1} T=\gamma_{2} T=1 / 100, \chi_{1}=-\chi_{2}=\chi$ (black solid line). Other parameters as in Fig. 11 Dashed horizontal line: $F_{\text {tot }}$ for the highest squeezed state in the lossless case. Inset: average number of atoms vs time for the same parameters.

narios even after a single loss event. In particular, for strongly asymmetric two-body losses one can protect superpositions by tuning the interaction energies, leading to useful states for high-precision atom interferometry applications. Such asymmetric losses occur in internal BJJs with ${ }^{87} \mathrm{Rb}$ atoms in the hyperfine states $\left|F, m_{F}\right\rangle=|2,-1\rangle$ and $|1,1\rangle$, subject respectively to fast two-body and slow one-body losses [16]. Finally, we note that the $\phi$ noise associated to the loss times $s_{K}$ could be used to obtain an indirect measurement of $s_{K}$ from the interferometric estimation of the phase rotation of the MSCSs, yielding to an observation of quantum jumps like in cavity QED [2].

\section{ACKNOWLEDGMENTS}

We are grateful to K. Rzążewski, F. Hekking, P. Treutlein, C. Gross, and M. Oberthaler for helpful discussions. D.S and K.P acknowledge support from the project ANR09-BLAN-0098-01, A.M. from the ERC Handy-Q project, and K.P. from the Polish Government Funds for the years 2010-2012 and the project "Decoherence in long range interacting quantum systems and devices" sponsored by the Baden-Württemberg Stiftung.

\section{Appendix A: Derivation of Eq. (9)}

Let us show that the quantum trajectory $t \mapsto\left|\psi_{1}(t)\right\rangle$ having one loss event in channel $m$ at the random time $s \in[0, t]$ is given by Eq. (91). We prove this formula for $m=1$ (the other cases $m=2$ and $m=12$ are similar). We first determine how an initial Fock state $\left|n_{1}, n_{2}\right\rangle$ is transformed if two atoms are lost in mode 1 at time $s$. Using Eq. (44), this state becomes $\sqrt{n_{1}\left(n_{1}-1\right)} e^{-\mathrm{i} \Phi_{t, s}\left(n_{1}, n_{2}\right)}\left|n_{1}-2, n_{2}\right\rangle$ at time $t$, where $\Phi_{t, s}\left(n_{1}, n_{2}\right)=(t-s) H_{\mathrm{eff}}\left(n_{1}-2, n_{2}\right)+s H_{\mathrm{eff}}\left(n_{1}, n_{2}\right)$ is a complex dynamical phase and $H_{\text {eff }}\left(n_{1}, n_{2}\right)$ are the quadratic eigenvalues of $\hat{H}_{\text {eff }}$. Setting $n_{2}=N_{0}-n_{1}$ yields

$$
\begin{aligned}
\Phi_{t, s}\left(n_{1}, n_{2}\right)= & t H_{\text {eff }}\left(n_{1}-2, n_{2}\right)+n_{1} \phi_{1}(s) \\
& +\mathrm{i} n_{1} \ln \left(\tan \left(\frac{\theta_{1}(s)}{2}\right)\right)
\end{aligned}
$$

up to $n_{1}$-independent constants, with $\theta_{1}(s)=$ $2 \arctan \left(e^{-s\left(2 \gamma_{1}-\gamma_{12}\right)}\right)$ and $\phi_{1}(s)=2 s\left(U_{1}-U_{12}\right)$. The two last terms in (A1) correspond respectively to the energy and damping changes due to the atom loss at time $s$. Replacing $\left|n_{1}, n_{2}\right\rangle$ in the Fock-state expansion of the initial CS [see Eq. (10)] by the above transformed state, we get Eq. (9).

\section{Appendix B: Density matrix in the subspace with} $\left(N_{0}-2 J\right)$ atoms

We now determine the conditional density matrix $\hat{\rho}_{N_{0}-2}(t)$ for a single loss event $(J=1)$ in the Fock basis. Using Eq. (4) we find the distribution

$$
\begin{aligned}
& p_{m}^{(t)}(s ; 1)=\gamma_{m} e^{-s G_{m}}\left[\cosh \left(s \delta_{m}\right)\right]^{N_{0}-2} \\
& \quad \times \| e^{-i t \hat{H}_{\text {eff }}}\left|N_{0}-2 ; \theta_{m}(s), 0\right\rangle \|^{2} \frac{N_{0}\left(N_{0}-1\right)}{4}
\end{aligned}
$$

with $G_{i}=\left(2 \gamma_{i}+\gamma_{12}\right) N_{0}-2 \gamma_{i}-2 \gamma_{12}, i=1,2$, and $G_{12}=\left(\gamma_{1}+\gamma_{2}+\gamma_{12}\right) N_{0}-2 \gamma_{1}-2 \gamma_{2}-\gamma_{12}$. Averaging over trajectories as in Eq. (6) yields

$$
\begin{aligned}
& \left\langle n_{1}, n_{2}\left|\hat{\rho}_{N_{0}-2}(t)\right| n_{1}^{\prime}, n_{2}^{\prime}\right\rangle \\
& \quad \propto \sum_{m} \gamma_{m} C_{m}\left(t ; n_{1}, n_{1}^{\prime}\right)\left\langle n_{1}, n_{2}\left|\widetilde{\rho}_{N_{0}-2}^{\text {(noloss) }}(t)\right| n_{1}^{\prime}, n_{2}^{\prime}\right\rangle,
\end{aligned}
$$

where $\widetilde{\rho}_{N_{0}-2}^{\text {(noloss) }}(t)=e^{-\mathrm{i} t \hat{H}_{\text {eff }}}\left|N_{0}-2 ; \frac{\pi}{2}, 0\right\rangle\left\langle N_{0}-2 ; \frac{\pi}{2}, 0\right| e^{\mathrm{i} t \hat{H}_{\text {eff }}^{\dagger}}$ is the conditional state having no loss in $[0, t]$ for an initial CS with $N_{0}-2$ atoms, and

$$
C_{m}\left(t ; n, n^{\prime}\right)=\frac{1-e^{-t\left[G_{m}+\delta_{m}\left(n+n^{\prime}-N_{0}+2\right)+2 \mathrm{i} \chi_{m}\left(n-n^{\prime}\right)\right]}}{G_{m}+\delta_{m}\left(n+n^{\prime}-N_{0}+2\right)+2 \mathrm{i} \chi_{m}\left(n-n^{\prime}\right)} .
$$

Equation (B1) shows that the density matrix conditioned to a single loss event is a superposition of CSs with $N_{0}-2$ atoms modulated by the envelope $\sum \gamma_{m} C_{m}\left(t ; n, n^{\prime}\right)$ and the damping factor of Eq. (77).

The above calculation can be generalized to trajectories with $J>1$ jumps between 0 and $t$. For $\gamma_{m} t \ll 1$ and $J \ll N_{0}, \hat{\rho}_{N_{0}-2 J}(t)$ is still given by Eq. (B1) upon replacing $\sum \gamma_{m} C_{m}\left(t ; n_{1}, n_{1}^{\prime}\right)$ by $\left[\sum_{m} \gamma_{m} C_{m}\left(t ; n_{1}, n_{1}^{\prime}\right)\right]^{J}$.

\section{Appendix C: Weak loss regime}

In this appendix we study the density matrix (B1) in the subspace with $N=N_{0}-2$ atoms in the weak loss regime $\gamma_{m} \ll q \chi / N_{0}$. 
One can show [27] that the density matrix $\hat{\rho}^{(0)}\left(t_{q}\right)$ associated with the superposition of CSs $\sum_{k} c_{k}\left|N ; \frac{\pi}{2}, \phi_{k}\right\rangle$ formed at time $t_{q}$ in the absence of losses has the following structure in the Fock basis: $\left\langle n_{1}, n_{2}\left|\hat{\rho}^{(0)}\left(t_{q}\right)\right| n_{1}^{\prime}, n_{2}^{\prime}\right\rangle$ is the sum of two matrices, the first one having zero matrix elements for $n_{1}^{\prime} \neq n_{1}$ modulo $q$ and corresponding to the statistical mixture of CSs $\sum_{k}\left|c_{k}\right|^{2}\left|N ; \frac{\pi}{2}, \phi_{k}\right\rangle\left\langle N ; \frac{\pi}{2}, \phi_{k}\right|$, and the second one having zero matrix elements for $n_{1}^{\prime}=n_{1}$ modulo $q$ and corresponding to the coherences $\sum_{k \neq k^{\prime}} c_{k} c_{k^{\prime}}^{*}\left|N ; \frac{\pi}{2}, \phi_{k}\right\rangle\left\langle N ; \frac{\pi}{2}, \phi_{k^{\prime}}\right|$.

For weak losses $\gamma_{m} \ll q \chi / N_{0}$ and equal interaction energies $U_{1}=U_{2}$,

$$
\left|C_{m}\left(t_{q} ; n, n^{\prime}\right)\right| \simeq \frac{\pi}{|\chi| q}\left|\operatorname{sinc}\left(\frac{\pi\left(n-n^{\prime}\right)}{q}\right)\right|
$$

vanishes at the values $n^{\prime}=n+p q(p= \pm 1, \pm 2, \cdots)$ corresponding to the nonzero off-diagonal elements of the aforementioned statistical mixture of CSs, and decays like $\left|n^{\prime}-n\right|^{-1}$ for the other off-diagonal elements encoding the coherences. Hence the statistical mixture of CSs is transformed after one loss event into a mixture of Fock states (complete phase relaxation). The inter-component coherences are, however, non vanishing, except for $\gamma_{1}=\gamma_{2}$ and $q=2$, because then the contributions of the two channels cancel each other, $\sum_{m} \gamma_{m} C_{m}\left(t_{2} ; n, n^{\prime}\right) \simeq 0$ for $n \neq n^{\prime}$. This explains the low and high values of $F_{N_{0}-2}\left(t_{2}\right)$ for symmetric and asymmetric losses seen in Fig. 2 (recall that the coherences are responsible for the quantum correlations quantified by the Fisher information).

Taking now asymmetric interaction energies, $\chi_{1}=0$ and $\chi_{2}=-2 \chi$, one infers from

$$
\sum_{m} \gamma_{m} C_{m}\left(t_{2} ; n, n^{\prime}\right) \simeq \frac{\pi}{2 \chi}\left(\gamma_{1}+\gamma_{2} \delta_{n n^{\prime}}\right)
$$

that $\hat{\rho}_{N_{0}-2}\left(t_{2}\right) \propto \widetilde{\rho}_{N_{0}-2}^{\text {(noloss) }}\left(t_{2}\right)$ when $\gamma_{2}=0$. Therefore, the conditional state is a two-component superposition slightly modified by Gaussian damping.
[1] D. Giulini et al., Decoherence and the Appearance of a Classical Word in Quantum Theory (Springer, Berlin, 1996)

[2] S. Haroche and J.-M. Raimond, Exploring the Quantum: Atoms, Cavities, and Photons (Oxford University Press, Oxford, 2006)

[3] B. Yurke and D. Stoler, Phys. Rev. Lett. 57, 13 (1986)

[4] U. Fano, Phys. Rev. 124, 1866 (1961)

[5] H. Feshbach, Annals of Physics (N.Y.) 5, 357 (1958)

[6] F. Piazza, L. Pezzé, and A. Smerzi, Phys. Rev. A 78, 051601 (2008)

[7] G. Ferrini, A. Minguzzi, and F.W.J. Hekking, Phys. Rev. A 78, 023606 (2008)

[8] J. Estève, C. Gross, A. Weller, S. Giovanazzi, and M. K. Oberthaler, Nature (London) 455, 1216 (2008)

[9] F. Riedel, P. Böhi, Y. Li, T. W. Hänsch, A. Sinatra, and P. Treutlein, Nature (London) 464, 1170 (2010)

[10] C. Gross, T. Zibold, E. Nicklas, J. Estève, and M. K. Oberthaler, Nature (London) 464, 1165 (2010)

[11] A. Sinatra and Y. Castin, Eur. Phys. J. D 4, 247 (1998); Y. Li, Y. Castin, and A. Sinatra, Phys. Rev. Lett. 100, 210401 (2008); Y. Li, P. Treutlein, J. Reichel, and A. Sinatra, Eur. Phys. J. B 68, 365 (2009)

[12] A. Sinatra, J.-C. Dornstetter, and Y. Castin, Front. Phys. 7, 86 (2012)

[13] K. Pawłowski and K. Rzążewski, Phys. Rev. A 81,
$013620(2010)$

[14] Y. P. Huang and M. G. Moore, Phys. Rev. A 73, 023606 (2006)

[15] G. Ferrini, D. Spehner, A. Minguzzi, and F.W.J. Hekking, Phys. Rev. A 82, 033621 (2010)

[16] C. Gross, J. Phys. B 45, 103001 (2012)

[17] K. Pawlowski, D. Spehner, A. Minguzzi, and G. Ferrini, in preparation

[18] G. J. Milburn, J. Corney, E. M. Wright, and D. F. Walls, Phys. Rev. A 55, 4318 (1997)

[19] J. Anglin, Phys. Rev. Lett. 79, 6 (1997)

[20] M. W. Jack, Phys. Rev. Lett. 89, 140402 (2002); M. W. Jack, Phys. Rev. A 67, 043612 (2003)

[21] H. Carmichael, An Open System Approach to Quantum Optics (Springer-Verlag, New York, 1991)

[22] J. Dalibard, Y. Castin, and K. Mølmer, Phys. Rev. Lett. 68, 580 (1992)

[23] An analogous result has been found in [12] for weak losses, i.e., without $\theta$ noise.

[24] S. L. Braunstein and C. M. Caves, Phys. Rev. Lett. 72, 3439 (1994)

[25] P. Hyllus, L. Pezzé, and A. Smerzi, Phys. Rev. Lett. 105, 120501 (2010)

[26] L. Pezzé and A. Smerzi, Phys. Rev. Lett. 102, 100401 (2009)

[27] G. Ferrini, D. Spehner, A. Minguzzi, and F.W.J. Hekking, Phys. Rev. A 84, 043628 (2011) 